\title{
Energy internet or comprehensive energy network?
}

\author{
Yusheng XUE (ه)
}

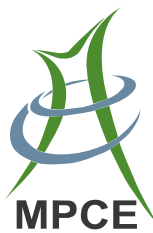

\begin{abstract}
What needs to be developed from the concept of "Smart Grid" is that: when renewable energy sources are absolutely prevailing in power generation, distributed power generation and distributed energy storage systems are widespread across the grid, and electric vehicle charging loads are prevailing in power load demands, how can the power grid support electric power as a core secondary energy source, undertake the role of a bridge between primary energy and end-use energy, and achieve the coordination and the optimization in macro energy perspective; how to guarantee the security of both macro energy and environment as well as the reliability of electricity. If a new term is needed, it should be Comprehensive Energy Network, not Energy Internet.
\end{abstract}

Keywords Energy security, Environmental security, Primary energy, Electric power, End-use energy, Smart grid, Energy internet, Comprehensive energy network

\section{Two replacements in energy flows}

Energy sources are important material basis of social development, while reliable power supply is a crucial support for modern civilization. Once the primary energy is being transformed into secondary energy in the form of electricity by various power stations, it will be delivered to various users through power transmission and distribution

CrossCheck date: 18 December 2014

Received: 18 December 2014/ Accepted: 6 March 2015/Published online: 24 March 2015

(C) The Author(s) 2015. This article is published with open access at Springerlink.com

Y. XUE, State Grid Electric Power Research Institute,

Nanjing 211000, China

$(\bowtie)$ e-mail: xueyusheng@sgepri.sgcc.com.cn networks as end-use energy. Herein, electric power is the core of secondary energy sources, while power grid is the physical platform for both wide-area electric power sharing and electric power market transactions. Therefore, it undertakes the role of a hub bridge between primary energy and end-use energy.

The pollution caused by human activities has brought additional loads into the cycle of natural ecosystem. The emission from each link of energy development and consumption chains has a serious impact on the safety margin of ecosystem. Once the ecological load goes beyond the selfrecovery capability of nature, ecological balance will be undermined and more natural disasters will inevitably strike the human society as a kind of punishment, including blackouts, even the irreversible destruction of human civilization [1].

Among all kinds of primary energy sources, fossil fuels remain the major resource for power generation at present. In 2013 , about $77.9 \%$ of electric power all over the world comes from fossil fuels [2]. However, it is not only threatened by the exhausting natural resources, but also restrained by environmental protection. Except for a few kinds of end-use consumptions that cannot be replaced by electric power from renewable energy sources, other enduse consumptions on fuels, power and so on can all be replaced [3].

According to the experience of developed countries, if the proportion of electric power in end-use energy increases by $1 \%$, the energy intensity will decrease by $3.7 \%$. It is estimated that the proportion of China's electric power in end-use energy will increase from $18.5 \%$ in 2009 to $28.0 \%$ in 2020 and $32.0 \%$ in 2030 [4]. The importance of electric power to energy security and environmental security is obvious.

In order to keep the reliability of energy resources and the stability of ecosystem within a controllable range, proper policies must be made and carried out strictly: 
improving the safety margin of nature through ecological engineering; replacing fossil energy sources with renewable energy sources in energy production; replacing fossil energy sources with electric power in end-use consumption, e.g. electric vehicles and smart cities. Meanwhile, energy conservation and emission reduction should be guided by both market mechanism and essential regulation.

\section{Relationship between power grid and macro energy network}

It is difficult to deliver renewable energy sources such as hydro, wind and solar energy in their original forms. And they can be consumed by end-use loads and shared in large area only after being transformed into electric power. Besides, the generation output of wind turbines and photovoltaic arrays are also intermittent and stochastic to a great extent. They cannot be directly controlled, and their forecast errors substantially exceed the forecast error of load. The time and the extent of their sudden changes are especially difficult to achieve precise forecast. Assuming that the renewable energy sources account for $50 \%$ or more among all resources for power generation, and the output of wind and solar power can decrease rapidly in a couple of minutes and keep very low for even hours, thus the electric power is a problem that should never be overlooked.

The dispatching problems of instantaneous reserve and spinning reserve of power system will be more and more outstanding with the increase of the proportion of renewable energy sources, and the security of secondary and enduse energies will be severely challenged. Without strong and reliable wide-area transmission grids, and intelligent and flexible initiative power distribution networks, it is impossible to replace fossil fuels with electric power on a large scale or coordinate the optimization of various primary, secondary and end-use energies.

In dealing with haze and pollution, the lowest total social cost should be set as the goal while the energy structure is being coordinated and improved, including replacing the fossil fuels with renewable energy sources and electric power in a larger scale, optimizing the structure and arrangement of fossil fuel development and utilization, and the transformation of secondary energy, and reforming the consumption mode of end-use energy.

In the link of energy delivery, the transmission of electric power, coal, natural gas as well as comprehensive transportation system should be correspondingly coordinated, and the transmission of electric power should be made full use of for achieving the thoroughfare of sharing renewables on a large scale through power grids [5]. With the surge of shale gas development, the amount and proportion of gas-fired power generation will be increased as well, while the power-to-gas technology will help with the integration of the intermittent excessive electric power [6]. These factors have strengthened the interaction of a gas transmission network, and a power grid in planning [7] and operating [8]. In addition, the thermal energy generated from distributed gas power generation and cogeneration has promoted the interconnection between heat supply networks and power distribution networks.

Aside from ensuring the security and adequacy of macro energy system, it is also necessary to study the reliability of capital, material, talent and information flow as well as the influence of policies and supervision so as to support the security, coordination and optimization of electric power flow and energy flow.

As an important platform of energy transmission, six extra-high voltage transmission lines have been put into operation by State Grid Corporation of China (SGCC). They are currently serving $20.02 \%$ of electric power transaction of the national power market and are estimated to be able to save 140 million tons standard coal in North, South and East China. The current trans-provincial and trans-regional transmission capacity of SGCC is about 1 $\mathrm{GW}$, which is yet far from the power transmission demand of $4.6 \mathrm{GW}$ in 2020. The hydropower of Tibet will be the main force of western China hydropower to be transmitted eastward, and the trans-regional transmission of solar power will be up to $0.01 \mathrm{GW}$ around 2030. Wind and solar power will be the main impetus of electric power flow, and extra-high voltage power grids will be the transmission platform for a larger area around 2050 [9].

A power system bearing such an important task must be able to withstand disturbance without collapse (i.e., stability) and keep balance between the supply and the demand anytime and anywhere after the disturbance (i.e., adequacy). The disturbance to be considered must not be limited within the power grid as at present, but extreme natural disasters, primary energy events and emergencies of other external links must be reasonably taken into account as well.

To attain these aims, major breakthroughs must be achieved in numerous relevant fields, which include but not limited to the following contents: technologies in various renewable energy power generation, wide-area power transmission network and initiative power distribution network, the forecast of wind and photovoltaic power generation on different time scales, energy storage, electric power replacement, configuration and dispatching of reserve capacity (including the maximum power and total energy it can provide) and information integration with energy systems [10].

Although China's blackout defense system has accomplished achievements so far, as its monitoring scope is limited within the power system, it cannot prevent blackouts 
caused by extreme natural disasters, serious primary energy events and other external factors. Therefore, the research scope must be expanded to the evolutionary process and mechanism of external disasters and the monitoring, and the prediction range must be moved forward to the source of external disasters so as to provide a longer early-warning time for blackout defense [11]. The lightning warning software developed by NARI has been running in the blackout defense system of Jiangxi power grid for 4 years and has achieved satisfying results, while the more comprehensive research and application are also in implementation [12].

\section{Confusion about energy internet}

To ensure energy security effectively, a top-level design is necessary. The U.S., European Union, Japan, and China have all mentioned the concept of Energy Internet [13-17]. What is an Energy Internet, after all? Currently the opinions vary, and might be more confusing in a considerably long period in the future. In many versions, Internet terms in information technology such as "IP address", "router" and "communication protocol", are directly borrowed into electric power flow or energy flow, while others mechanically apply the descriptions used in smart grid.

Internet is a kind of computer technology for supporting information flow. It refers to a network built on a bunch of communication protocols for connecting different computers with a common language, thus it can achieve the communication between computers. For a specific process of information exchange, specific receivers can be assigned. Its delivery paths can be scheduled. It can be buffered, saved or delayed for delivery.

However, electric power flow follows the physical law which is different from that of information flow. The electric power injected by power generation units (including discharging batteries) and the electric power consumed by electrical load (including power losses and charging batteries) in a power grid are sure to be balanced in any moment. The electric power flow follows the Kirchhoff Law, in which the input and the output on each electrical node are sure to be balanced in any time section, without any possibility of buffering, saving or delaying. Even under the abnormal condition in power grid, the balanced relations mentioned above still exist; only the balance will be brought to collapse with the decrease of electric energy quality, equipment overload, forced load shedding or disconnection of power grids. The unbalance of power will only occur at generator rotors or motor rotors; the unbalance between its mechanical power and electromagnetic power will result in various electromechanical stability problems.

Generally, "specific users purchase the electric energy of specified power plants" can only be understood from the perspective of market clearing. At the physical level, all power units inject electric power into the connected grid while all the electricity users consume electric power from the grid they are connected to. Theoretically, it is impossible to identify the information that to which users the electric power a power plant injects into the power grid are flowing, or from which power plant the electric power consumed by a certain user is delivered.

Energy Internet composed of physical systems must obtain the wide support from information network. Both of them must be integrated into each other, otherwise everything will be wishful thinking. The information flow comprehensively supports the security and optimization of the whole energy flow, but the concept of Internet cannot be applied mechanically to Energy Internet. Describing the Energy Internet as similar to Internet or simply integrating the power grid and the communication network does no good to the development of technology,thus it can only lead to more confusion.

On the other hand, if the concepts of Energy Internet and Smart Grid cannot be clearly distinguished clearly if all the descriptions about Energy Internet have repeatedly appeared in literatures of Smart Grid early on, why should we put forward new terms for it?

\section{Comprehensive energy network}

At present, the academic community has achieved some certain consensus on the concept of Smart Grid. If it is taken as our vision in future power system, the new expectation of power system can also be incorporated into this definition without a new term.

If new concepts are to be put forward in respect of the interaction with various external non- electric- powersystems, one convincing reason will be that, in order to ensure the security of such a core link of energy sources as electric power, the upstream and downstream in electric power flow must be studied at the level of macro energy systems, i.e., the interactions between electric energy, primary energy and end-use energy, as shown in Fig. 1.

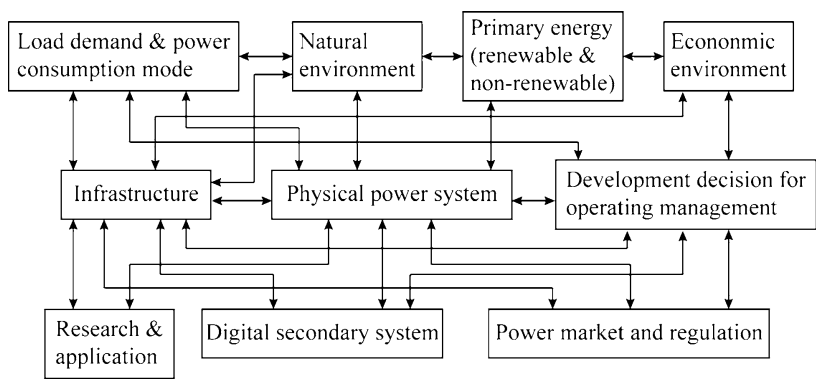

Fig. 1 Interactions between power system and its external environment 
Herein, what is to be discussed about power system is that, when renewable energy sources account for $80 \%$ or more in power system; renewable energy sources account for $80 \%$ or more in long distance power transmission; distributed generation and energy storage systems are widely applied [18, 19], and the charging loads of electric vehicles account for $30 \%$ or more on power load [20], what new denotations are required for the present concept of smart grid [21]? What influences may be brought about by the breakthroughs of energy conversion and storage technology, and by what means can power grid adapt to the revolution of macro energy?

Disturbances of external factors in power system, such as natural, economic and social environments, other energy systems, human talent, innovation, infrastructure, policies, regulation, and other links, may affect the reliable operation of power system. Conversely, the security and the stability of power system will affect the operation of each external link as well. Therefore, the interactions between power system and external links must be taken into consideration, while the adequacy and security issues must be studied at the level of large energy sources [22].

Figure 2 shows the whole process of energy production and consumption, in which the red part denotes the core of secondary energy sources. It is obvious that the electric power has a critical role to play in connecting the primary energy and end-use energy.

The top-level design of energy management in this paper is the comprehensive interaction and coordinated optimization among various primary energies including renewable energy and the secondary energy dominated by electric power and various end-use energies in demand side. Thus, I prefer to call it "Interconnected Energy Network" or "Comprehensive Energy Network".

Even if the name "Energy Internet" ("Neng Yuan Hu Lian Wang" in Chinese) is followed due to custom or other reasons, it should be understood as "Macro Energy Network", and the corresponding English name should be "Comprehensive Energy Network", not "Energy Internet".

\section{Conclusions}

The sustainable development of human beings not only depends on energy sources, but also on economic security and environmental security, which interact closely and influence each other. In electric power industry, comprehensive energy security based on the security of power systems should be emphasized. It is closely related with the revolution of primary and end-use energies, and must be treated with the concept of macro- energy perspective.

Smart Grid has clarified the idea of in-depth integration of information and electric power flow, while further expansion should be guided by the concept of macro energy perspective, which means to extend from the internal of a power system to relevant non-power-systems. It is necessary to study the influence of various non-power-system links on both reliability and economy of power systems, avoiding unwanted winding courses for the development of power systems and markets as well as preventing serious large-region blackouts caused by extreme events, which is difficult to be recovered in a reasonable time period. Meanwhile, how can the power systems promote the

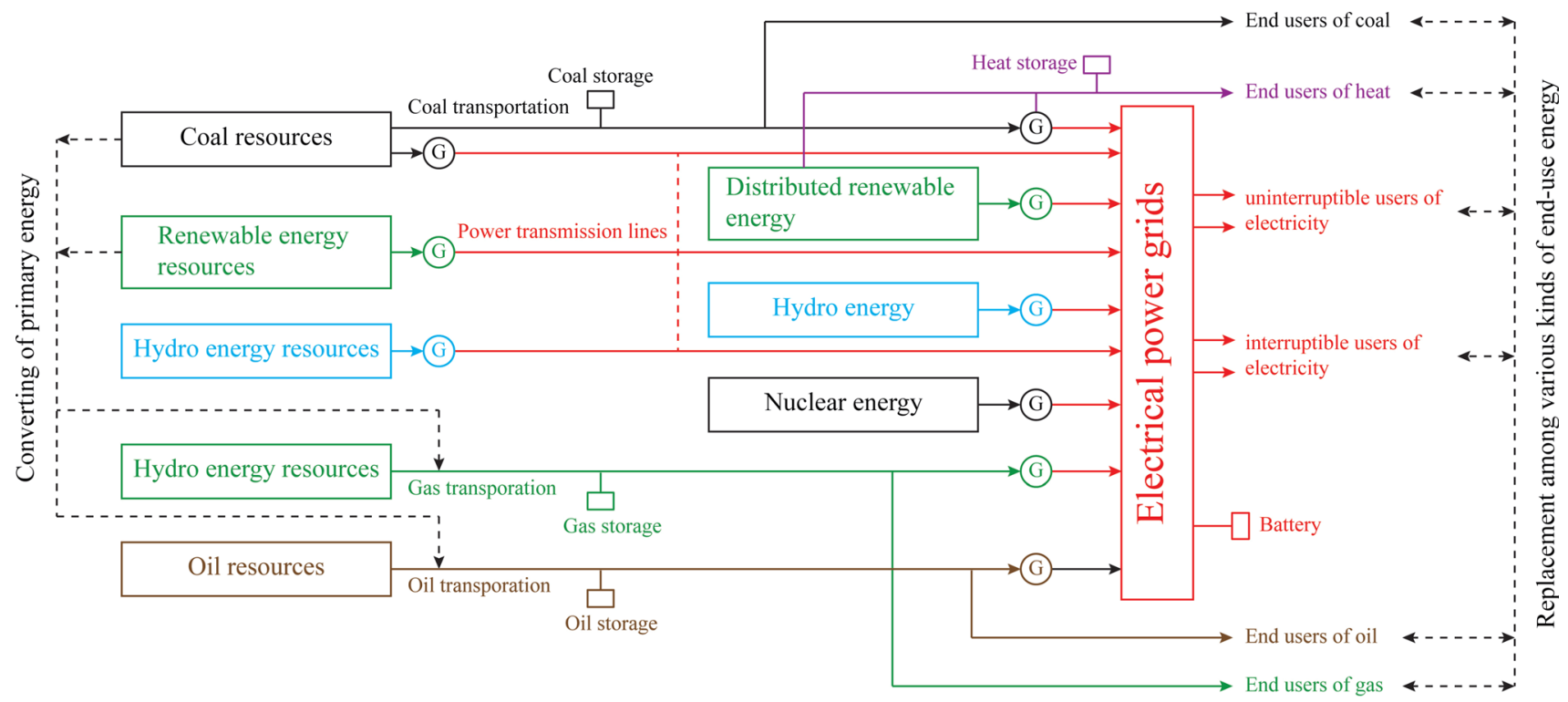

Fig. 2 Process of energy production and consumption 
security of both environments and macro energy sources should be studied as well.

Acknowledgments This work is supported by National High Technology Research and Development Program of China (863 Program) (No. 2011AA05A105) and SGCC Projects.

Open Access This article is distributed under the terms of the Creative Commons Attribution License which permits any use, distribution, and reproduction in any medium, provided the original author(s) and the source are credited.

\section{References}

[1] Climate change 2013 synthesis report (2013) Summary for policymakers. Intergovernmental Panel on Climate Change (IPCC), Geneva, Switzerland

[2] Renewables 2014 global status report (2014) Renewable energy policy network for the 21st century (REN21), Paris, France

[3] Xue YS, Cai B, James G et al (2014) Primary energy congestion of power systems. J Mod Power Syst Clean Energy 2(1):39-49

[4] Liu ZY (2012) Electric power and energy in China. China Electric Power Press, Beijing (in Chinese)

[5] Almassalkhi M, Hiskens I (2011) Optimization framework for the analysis of large-scale networks of energy hubs. In: Proceedings of the 17 th power system computation conference (PSCC'11), Stockholm, Sweden, 22-26 Aug 2011, 7 pp

[6] World's largest power-to-gas plant for generating methane enters operation (2012) Zentrum für Sonnenenergie-und WasserstoffForschung Baden-Württemberg (ZSW), Baden- Württemberg, Germany

[7] Saldarriaga CA, Hincapié RA, Salazar H (2013) A holistic approach for planning natural gas and electricity distribution networks. IEEE Trans Power Syst 28(4):4052-4063

[8] Krause T, Andersson G, Fröhlich K et al (2011) Multiple-energy carriers: modeling of production, delivery, and consumption. Proc IEEE 99(1):15-27

[9] Bai JH (2013) The UHV interregional transmission should be more rapidly developed on a large scale. http://www.indaa.com. cn/pl2011/zxpl/201411/t20141113_1579299.html. Accessed 13 Nov 2013 (in Chinese)

[10] Zhao JH, Wen FS, Xue YS et al (2011) Modeling analysis and control research framework of cyber physical power systems. Automat Electr Power Syst 35(16):1-8 (in Chinese)
[11] Xue YS, Wu YJ, Xie YY et al (2013) Extension of blackout defense scheme to natural disasters early-warning. Automat Electr Power Syst 37(16):18-26 (in Chinese)

[12] Xie YY, Xue YS, Wang HH et al (2013) Space-time earlywarning of power grid fault probability by lightning. Automat Electr Power Syst 37(17):44-51 (in Chinese)

[13] Rifkin J (2011) The third industrial revolution: How lateral power is transforming energy, the economy, and the world. Palgrave MacMillan, New York

[14] Recorded conference "Mission growth: Europe at the lead of the new industrial revolution". European Commission. http://ec.europa. eu/avservices/video/player.cfm?ref=85716. Accessed 29 May 2012

[15] E-energy. Federal Ministry of Economics and Energy of Germany. http://www.e-energy.de/en/index.php

[16] Huang AQ, Crow ML, Heydt GT et al (2011) The future renewable electric energy delivery and management (FREEDM) system: the energy internet. Proc IEEE 99(1):133-148

[17] Dong ZY, Zhao JH, Wen FS et al (2014) From smart grid to energy internet: basic concept and research framework. Automat Electr Power Syst 38(15):1-11 (in Chinese)

[18] Basua AK, Chowdhuryb SP, Chowdhuryb S et al (2011) Microgrids: energy management by strategic deployment of DERs - a comprehensive review. Renew Sustain Energ Rev 15(9):4348-4356

[19] Wang CS, Li P (2010) Development and challenges of distributed generation, the microgrid and smart distribution system. Automat Electr Power Syst 34(2):10-14 (in Chinese)

[20] Wang GB, Zhao JH, Wen FS et al (2012) Stochastic optimization dispatching of plug-in hybrid electric vehicles in coordination with renewable generation in distribution systems. Automat Electr Power Syst 36(19):22-29 (in Chinese)

[21] Xue YS, Li TR, Yin X et al (2013) Managements of generalized congestions. IEEE Trans Smart Grid 4(3):1675-1683

[22] Xue YS, Xiao SJ (2013) Generalized congestion of power systems: Insights from the massive blackouts in India. J Mod Power Syst Clean Energ 1(2):91-100

Yusheng XUE received Ph.D. in Electrical Engineering from the University of Liege (Belgium) in 1987. He became a Member of Chinese Academy of Engineering in 1995. He is now the Honorary President of State Grid Electric Power Research Institute (SGEPRI). His research interests include nonlinear stability, control and power system automation. 